\title{
Galaxy evolution in Abell 85
}

\section{Cluster substructure and environmental effects on the blue galaxy population}

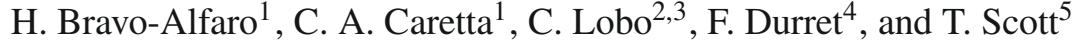 \\ 1 Departamento de Astronomía, Universidad de Guanajuato, Apdo. Postal 144, Guanajuato 36000, Mexico \\ e-mail: hector@astro.ugto.mx \\ 2 Centro de Astrofísica da Universidade do Porto, Rua das Estrelas 4150-762 Porto, Portugal \\ 3 Depto. de Matemática Aplicada, Faculdade de Ciências, Univ. do Porto, R. do Campo Alegre 687, 4169-007 Porto, Portugal \\ 4 Institut d'Astrophysique de Paris, CNRS, UMR 7095, Université Pierre et Marie Curie, 98bis Bd Arago, 75014 Paris, France \\ 5 Center for Astrophysics Research, University of Hertfordshire, College Lane, Hatfield, AL10 9AB, UK
}

Received 1 August 2008 / Accepted 25 September 2008

\begin{abstract}
In this series of papers we explore the evolution of late-type galaxies in the rich cluster Abell 85. In this first paper we revisit the complex dynamical state of A 85 by using independent methods. First, we analyze the galaxy redshift distribution towards A 85 in the whole range $0-40000 \mathrm{~km} \mathrm{~s}^{-1}$, and determine the mean redshifts of the background clusters A 87 and A 89, very close in projection to A 85. Then we search for substructures in A 85 by considering the 2D galaxy distribution of its members $\left(13000-20000 \mathrm{~km} \mathrm{~s}^{-1}\right)$ and by applying the kinematical 3D $\Delta$-test to both projected positions and radial velocities. This clearly reveals several substructures: one close to the cluster core and three more projected towards the southeast, along the region where an X-ray filament has been extensively studied. We also analyse the distribution of the brightest blue galaxies across a major fraction of the cluster volume, considering if they are gas-rich or poor. We report a very asymmetric distribution of the blue member galaxies, with most of them to the east and southeast, namely in the region joining the core of A 85 to its farthest substructure in this direction - dubbed the $S E$ clump. By matching our sample of bright blue member galaxies with $\mathrm{H}$ I detections reported in the literature, we identify gas-rich and gas-poor ones. As expected, the H I-rich blue galaxies follow the same trend as the parent sample, with most of them projected on the eastern side of the cluster as well. Interestingly no blue objects have been detected in H I up to a projected radius of $2 \mathrm{Mpc}$ in this zone. We finally estimate the ram pressure stripping exerted by the intra-cluster medium as a function of the projected distance from A 85, in order to quantify how important this mechanism might be in sweeping the gas out of the infalling spirals.
\end{abstract}

Key words. galaxies: evolution - galaxies: clusters: individual: Abell 85, Abell 87

\section{Introduction}

Important evolution is known to occur between $z \sim 1$ and the present time. Galaxy clusters keep evolving throughout these epochs by accreting galaxies and groups of galaxies, mainly along filamentary structures (e.g. Adami et al. 2005, and references therein), which gives rise to merging episodes in a hierarchical fashion. Many clusters display virialized cores surrounded by infall regions of galaxies and/or groups, which are gravitationally bound but not in equilibrium with the main cluster body. The presence (or absence) of such substructures is relevant since it provides tight constraints on models of structure formation and evolution at large scales. Important changes are also evident for individual galaxies: the cosmic star formation rate may suffer a change of an order of magnitude since $z=1$ (e.g. Lilly et al. 1996; Madau et al. 1996). Further major evidence of galaxy evolution is the decreasing number of spirals as one goes from the field to the central regions of clusters - and the corresponding rising population of lenticulars - in the nearby Universe (the morphology-density relation, Oemler 1974; Dressler 1980, 2004, and references therein).

Nowadays an interesting debate has arisen about this last topic: while some authors discard major environmental effects on structural parameters such as size and mass concentration (Li et al. 2006), extensive multifrequency surveys over large volumes of galaxy clusters have, on the other hand, provided new insights into the environmental effects on both star formation activity and morphology of individual galaxies (see for example Cayatte et al. 1990; van Gorkom 2004; Vollmer et al. 2004; Chung et al. 2007). Furthermore, the presence of groups of $\mathrm{E}+\mathrm{A}$ galaxies in very specific regions of clusters like Coma strongly suggests that they have suffered recent - and structuralenvironmental processing.

In this work we revisit the dynamical state of Abell 85 (hereafter A 85) and the strength of ram pressure stripping to produce the gas deficiency observed to large distances from the cluster center. In a forthcoming paper (Paper II) we will study the possible gravitational effects exerted on individual galaxies infalling from the SE, by considering their gas content, activity features and stellar morphology seen in NIR. The goal of this series of papers is to explore the transformation of late-type galaxies as a function of the cluster environment under different scenarios such as effects produced by the intra cluster medium (ICM) and/or gravitational preprocessing.

The richness class 1 cluster A 85, located at redshift $z=$ 0.055 , is a very interesting system that provides the perfect laboratory for studying the effects of environment on the galaxy population and on the properties of the galaxies themselves (gas content, SFR, AGN activity, etc.). This cluster has been extensively studied in the radio continuum, optical and X-rays (e.g. 
Durret et al. 1998b; Giovannini et al. 1999; Kempner et al. 2002; Durret et al. 2005). Many works have revealed a dynamically young system undergoing several mergings. For example, Kempner et al. (2002) proposed the existence of two subclusters around the inner part of A 85, and studied one of them based on Chandra observations; they report a subcluster much less massive than A 85, merging with the latter from the south. Two other Abell clusters, A 87 and A 89, are very close in projection to A 85 (Abell et al. 1989). Durret et al. (1998b) concluded that A 89 is rather constituted by two sheets of galaxies on the background, while A 87 would not be a separate cluster but rather a series of individual groups moving into the main body of A 85 from the SE. The global cluster orientation, as traced by X-rays and by the optical galaxy distribution, indicates an elongation towards the A 87 region, at a position angle of $160^{\circ}$, coincident with the large scale structure in which the cluster is embedded. This elongation also coincides with the orientation of the southern X-ray filament seen in different surveys.

We follow a systematic strategy to detect substructures around A 85 based on a thorough analysis applied to a large set of optical photometric and spectroscopic data (described in Sect. 2). Our first approach is based on revisiting the galaxy redshift distribution in the direction of A 85, up to $40000 \mathrm{~km} \mathrm{~s}^{-1}$ $(z \sim 0.133)$, and identifying the different systems along the line of sight (Sect. 3.1). We then probe, in Sect. 3.2, the 2D spatial distribution of the member galaxies of A 85 in the same field of view. For a better assessment of three-dimensional substructures, we perform a 3D kinematical analysis of the cluster making use of the $\Delta$-test (Dressler \& Shectman 1988), which is one of the most sensitive algorithms to trace substructures. This is described in Sect. 3.3. As a complementary method for probing substructures, we carry out an analysis of the distribution of the brightest blue galaxies in A 85 (in Sect. 3.4), considering if they are $\mathrm{H}$ I-rich or poor by matching positions with $\mathrm{H}$ I detections reported previously in the literature. We discuss in Sect. 4 the results yielded by the different methods and trace a scenario of galaxy evolution in A 85 where ram pressure seems to have played an important role in the evolution of the bright blue galaxies. This is supported by our estimates of the strength of ram pressure stripping as a function of the projected distance from the cluster center. We summarize our results in Sect. 5.

Throughout this paper we assume $\Omega_{\mathrm{M}}=0.3, \Omega_{\Lambda}=0.7$, and $H_{0}=75 \mathrm{~km} \mathrm{~s}^{-1} \mathrm{Mpc}^{-1}$. In this cosmology, the scale is $10^{\prime}=$ $0.6 \mathrm{Mpc}$ at the cluster mean redshift.

\section{Observational data}

We obtained position and optical magnitude data for galaxies in the A 85/87/89 complex from the SuperCOSMOS Science Archive, available from the Royal Observatory of Edinburgh ${ }^{1}$. The area covers a region of 50 arcmin radius around the center of A 85, taken as the position of its cD galaxy, MCG-0202-086, which also coincides with the peak of the X-ray emission of the cluster. Magnitudes in $b_{\mathrm{J}}$ and $r_{\mathrm{F}}$ bands came from the digitization of UKST Survey plates (IIIa-J + GG395 and IIIa-F + OG590, respectively) by the SuperCOSMOS machine, using $10 \mu \mathrm{m}$ size pixels (0.67") (Hambly et al. 2001b). These magnitudes were placed on an absolute scale using SDSS-EDR (Stoughton et al. 2002), EIS (Arnouts et al. 2001) and ESOSculptor Survey (Arnouts et al. 1997) data, and are good to about $0.1 \mathrm{mag}$ (rms). Note that a new calibration has been applied that supersedes the original SuperCOSMOS Sky Survey.

${ }^{1}$ http://surveys.roe.ac.uk/ssa/index.html
The detection limit of SuperCOSMOS data is estimated to be about $b_{\mathrm{J}}=20.5$ and $r_{\mathrm{F}}=19.5$, at a completeness level above $95 \%$, while the astrometry is accurate to better than $0.3^{\prime \prime}$ in both right ascension and declination (Hambly et al. 2001a).

This photometric catalogue was then matched with radial velocity data from the compilation of Andernach et al. $(2005)^{2}$, the largest compilation of velocities for Abell cluster member galaxies to date. For the A 85/87/89 complex (the area inside a radius of 50 arcmin around the center of A 85) this compilation contains 1693 entries, including multiple observations for most of the members. After discarding measurements with large deviations we averaged the radial velocities for each galaxy. This resulted in redshifts for 574 galaxies in the area of the A $85 / 87 / 89$ complex in the range $0-40000 \mathrm{~km} \mathrm{~s}^{-1}$. In the range 13000-20000 $\mathrm{km} \mathrm{s}^{-1}$, corresponding to A 85 (e.g. Durret et al. 1998a), the compilation yields 367 redshifts for member galaxies. We found a match of $98.9 \%$ between these galaxies and SuperCOSMOS data.

In order to study the global HI content in A 85 we consider the positions of twelve H I-detected galaxies in this cluster recently reported by Bravo-Alfaro et al. (2008). This was obtained from an $\mathrm{HI}$ survey of a region of about $1.5 \times$ 1.5 degrees centered on A 85, observed with the VLA in its C-configuration, pointing the array at multiple positions and central frequencies. In this fashion the whole complex A 85/87/89 has been homogeneously surveyed with an average noise of rms $\sim 0.25 \mathrm{mJy}$ beam $^{-1}$, within the velocity range 14580-18440 $\mathrm{km} \mathrm{s}^{-1}$. These observations have, on average, an $\mathrm{H}$ I mass detection threshold of $7 \times 10^{8} M_{\odot}$, corresponding to a $6 \sigma$ detection level. More details on the observations, on the complex data reduction process, and the gaseous properties of individual galaxies will soon appear (van Gorkom et al., in prep.).

\section{Results on the galaxy distribution}

\subsection{The velocity distribution towards $A 85$}

We revisit the velocity distribution in the direction of A 85 by analyzing the redshift catalogue compiled in Sect. 2. The distribution of these radial velocities is shown in Fig. 1. As expected there are many concentrations of galaxies along this line of sight. The most remarkable one is the A 85 spike, within the velocity range $13000-20000 \mathrm{~km} \mathrm{~s}^{-1}$. This peak corresponds to $64 \%$ of the galaxies in the cone. We find a biweight mean radial velocity of $16583(+60,-52) \mathrm{km} \mathrm{s}^{-1}$ and a velocity dispersion of 1122 $(+54,-54) \mathrm{km} \mathrm{s}^{-1}$ for the 367 member galaxies in this system, using a robust statistical (ROSTAT) estimation (Beers et al. 1990). Their velocity distribution, enlarged in Fig. 2, is not far from a Gaussian. It shows a small positive skewness of 0.11 , probably because of the small sub-peak between 19000 and $20000 \mathrm{~km} \mathrm{~s}^{-1}$. Most of the galaxies in this velocity sub-peak have positions coincident with a subcluster located in the western region of $\mathrm{A} 85$, that may be infalling into the main cluster. The kurtosis of the distribution is also positive (0.49), revealing a slightly peaked shape.

The next two prominent peaks along the line of sight to A 85, at 23500 and $28500 \mathrm{~km} \mathrm{~s}^{-1}$ respectively, whose centers are projected in the direction of A 89 (especially the former one), refer to the near and remote sheets identified by Durret et al. (1998b). Our sample consists of 77 and 48 galaxies for these

2 Most of the data for A 85 in this compilation comes from Beers et al. (1991); Malumuth et al. (1992); Durret et al. (1998a); Christlein \& Zabludoff (2003); Smith et al. (2004) and SDSS (Stoughton et al. 2002). 


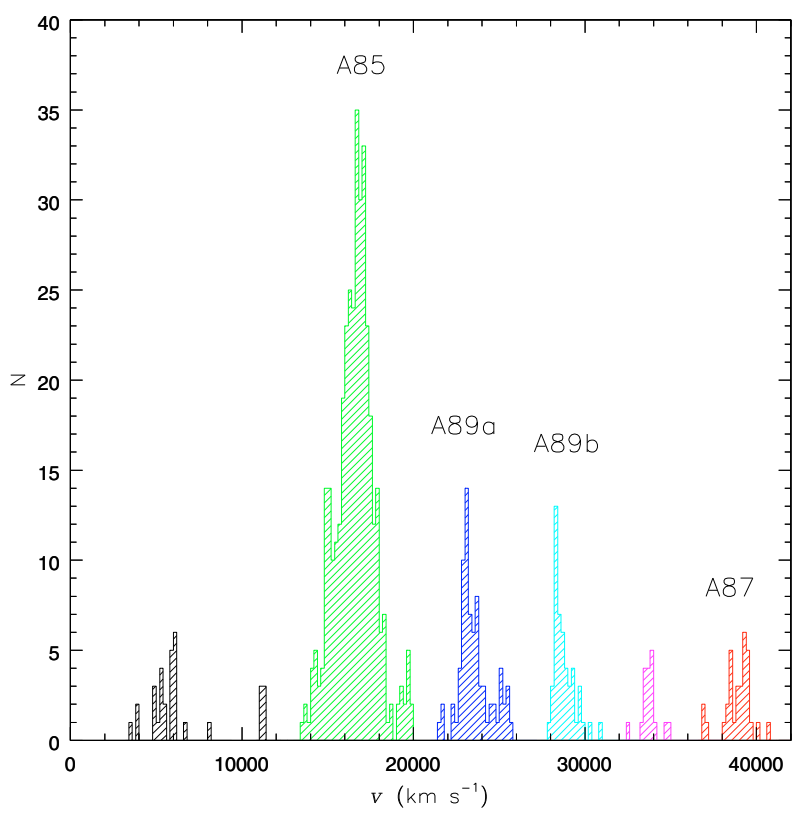

Fig. 1. Histogram showing the distribution of 574 velocities in the region of the A 85/87/89 complex. The most conspicuous concentrations are marked according to their cluster and/or group association.

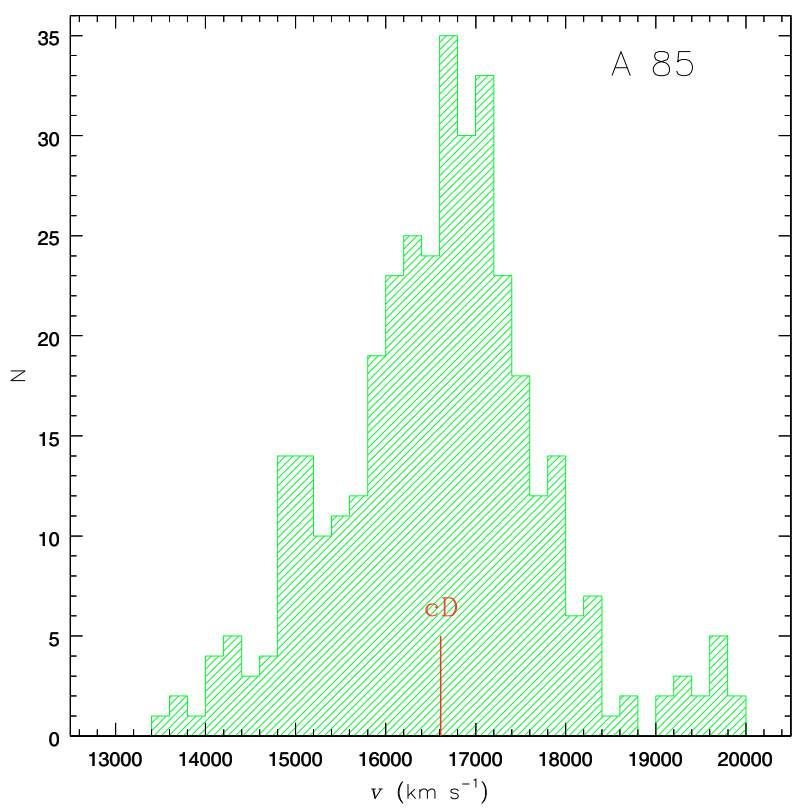

Fig. 2. Histogram showing the velocity distribution of galaxy-members of $\mathrm{A} 85$, within the range $13000-20000 \mathrm{~km} \mathrm{~s}^{-1}$.

two structures, respectively. The last peak $\left(\sim 39000 \mathrm{~km} \mathrm{~s}^{-1}\right)$ is centered on the A 87 region. This is most probably the system of galaxies associated with the A 87 cluster identified by Abell et al. (1989). A 87 is a distance class 5 cluster, which, following Abell's estimation, corresponds to about $39000 \mathrm{~km} \mathrm{~s}^{-1}$ on average $(z \sim 0.131)$. With 32 member galaxies, the ROSTAT calculation gives a biweight mean of $39014(+113,-143) \mathrm{km} \mathrm{s}^{-1}$ and a velocity dispersion of $737(+259,-110) \mathrm{km} \mathrm{s}^{-1}$ for this system of galaxies.

In general, our values of central velocity and velocity dispersion are in good agreement with recent works, for instance that of Christlein \& Zabludoff (2003). In spite of a slight difference in the velocity range we consider (13000-20000 $\mathrm{km} \mathrm{s}^{-1}$

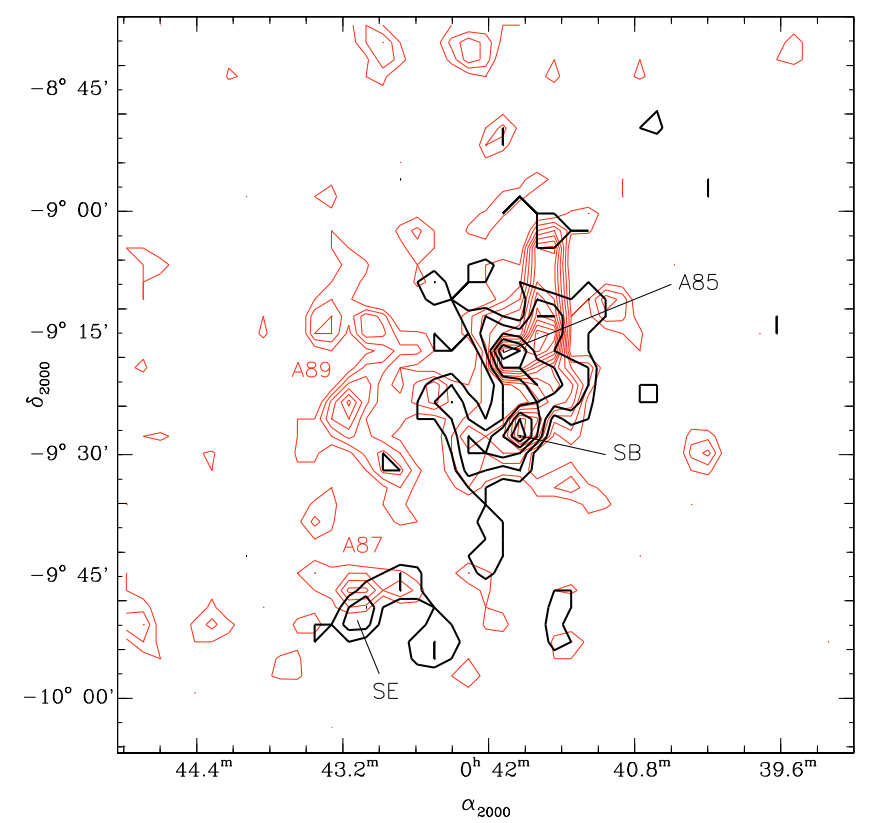

Fig. 3. Iso-density contours drawing the distribution of galaxies in the direction of A 85; red thin contours in the background correspond to all galaxies with magnitudes $b_{\mathrm{J}}<20.5$. Black thick contours indicate the distribution of the 367 members of A 85 (13000-20000 km s${ }^{-1}$ ). Main clusters and substructures of A 85 are indicated.

against $13423-19737 \mathrm{~km} \mathrm{~s}^{-1}$ in their work), our systemic velocity $\left(16583 \mathrm{~km} \mathrm{~s}^{-1}\right)$ is consistent with theirs $\left(16607 \mathrm{~km} \mathrm{~s}^{-1}\right)$. Instead, a small difference is seen between our reported velocity dispersion (1122 $\mathrm{km} \mathrm{s}^{-1}$ ) and Christlein \& Zabludoff's value (993 $\mathrm{km} \mathrm{s}^{-1}$ ), likely due to our larger velocity range and sample of galaxies (367 in this work against 280 in theirs).

\subsection{The $2 D$ galaxy distribution in $A 85$}

To better understand the structure of A 85 we constructed an isodensity contour map for the cluster members, i.e., those 367 galaxies with radial velocities in the range $13000-20000 \mathrm{~km} \mathrm{~s}^{-1}$. This is shown in Fig. 3 with black contours. The galaxies were counted within square cells of $4.1^{\prime}$ side, each cell displaced only half of its size from the previous one in order to smooth the contours. This provides a resolution of about $0.125 \mathrm{Mpc}$ at the distance of A 85. The plotted contours correspond to 3, 5, 7, 9 and 11 galaxies per cell (the lowest level corresponds to 0.2 galaxies/ $\operatorname{arcmin}^{2}$, while the peak reaches 0.8 galaxies/arcmin ${ }^{2}$ ). These contours are superimposed on the isodensity map of all galaxies with magnitudes $b_{\mathrm{J}}<20.5$ in the A 85/87/89 complex area (red contours). The A 89 concentration appears only in red contours, whereas the A 87 region presents a superposition of two structures. These structures are the A 87 cluster (at $39000 \mathrm{~km} \mathrm{~s}^{-1}$, seen in red contours) and, slightly displaced to the south, an infalling subcluster of A 85 (seen in black contours and labeled $S E$ in Fig. 3), the latter coincident with one of the clumps found by Durret et al. (1998b) in the filament region.

The black isodensity contours constitute the first galaxy density map of A 85 built exclusively with galaxy members. It reveals three significant peaks: the first represents the core of the main cluster (indicated with $A 85$ in the figure), nearly coincident with the CD galaxy; a secondary peak is coincident with the Southern Blob (SB as in Kempner et al. 2002); and a third, and less conspicuous one, appears some $35^{\prime}$ (about $2.1 \mathrm{Mpc}$ ) to the 


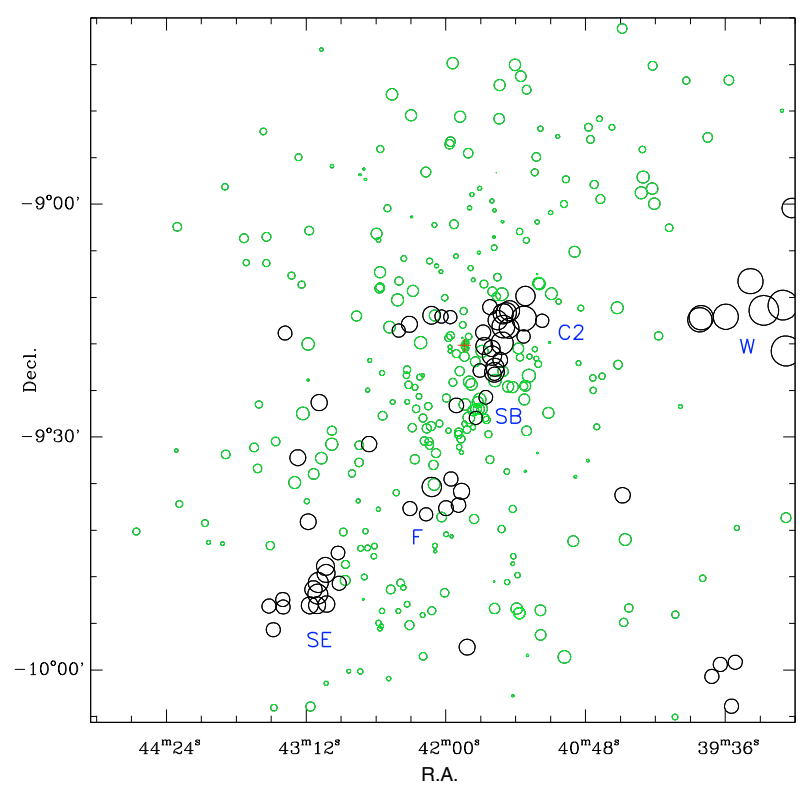

Fig. 4. Substructures detected around A 85 following the kinematical analysis of the $\delta$ parameter. Larger black circles indicate higher probabilities of the presence of substructures. The most conspicuous substructures detected are indicated. The red cross marks the cluster center, coincident with the $\mathrm{cD}$.

south-east of the cluster center (indicated as $S E$ in the figure), which corresponds to the subcluster superposed on A 87 already mentioned above. We analyze in more detail this $S E$ subcluster in the next section.

\subsection{The $3 D \Delta$-test}

As pointed above the galaxy density map of A 85 members with velocities between 13000 and $20000 \mathrm{~km} \mathrm{~s}^{-1}$ already shows a non-uniform distribution. Now we move a step forward in quantifying the presence of substructures by applying a more powerful statistical test that considers both positions and velocities. One of the most sensitive algorithms proposed in the literature (e.g. Pinkney et al. 1996) is the $\Delta$-test proposed by Dressler \& Shectman (1988), which searches for deviations of the local velocity mean and dispersion from the global values. These statistics produce a deviation measure ( $\delta$ parameter) for each galaxy, which can be used to find subclumps of "deviant" galaxies:

$\delta^{2}=\frac{\left(N_{\text {neighb }}+1\right)}{\sigma^{2}}\left[\left(v_{\text {local }}-v\right)^{2}+\left(\sigma_{\text {local }}-\sigma\right)^{2}\right]$

where $N_{\text {neighb }}$ is the number of neighbors used to estimate the local mean velocity $\left(v_{\text {local }}\right)$ and velocity dispersion $\left(\sigma_{\text {local }}\right)$, while $v$ and $\sigma$ are the global mean velocity and velocity dispersion, respectively.

A correlated spatial and kinematic variation, i.e., the presence of a region (RA, Dec) containing several galaxies that deviate from the global kinematic values, indicates a high probability of substructure in this zone. In the following discussion we indicate these clumps of galaxies as showing high kinematical deviations from the global behavior. The cumulative value of $\delta$ for the entire cluster $(\Delta)$ can be compared to Monte Carlo simulations to estimate the confidence level of the general deviation of the cluster.

We applied the $\Delta$ statistics to the 367 members of A 85, using $N_{\text {neighb }}=10$, and the corresponding results are shown in Fig. 4 (we obtained similar solutions with values of $N_{\text {neighb }}=15$,
$19 \simeq \sqrt{N}$, and $37 \simeq N / 10)$. In Fig. 4 each galaxy is marked by a circle with a size proportional to $\delta$ (instead of $e^{\delta}$, as usual, since the signal in A 85 is very high). We take the arbitrary value $\delta=2.0$ as a convenient threshold to discriminate objects with kinematics that deviate significantly from the cluster one. Therefore, galaxies with $\delta>2.0$ are indicated with black circles in Fig. 4, while green circles represent galaxies with $\delta<2.0$. The cumulative $\Delta$ parameter for the cluster is larger than the one obtained in $99.3 \%$ of 1000 Monte Carlo random simulations.

We note five prominent regions displaying enhanced variation in kinematics with respect to the mean; these are spots of larger black circles in Fig. 4: the first one is close to the center, a few arcmin to the west, and we identify it as $C 2$. Another substructure is found towards the south, coincident with the Southern Blob $(S B)$, and two more appear in the southeast region of A 85: one along the X-ray filament detected in previous works, namely with ROSAT and XMM-Newton data $(F$, see Durret et al. 2003, and references therein), and the other along an extension of the latter (indicated with $S E$ ). We detect a last substructure to the west $(W)$ of the cluster.

\subsection{The distribution of the brightest blue galaxies in $A 85$}

Another effective method to trace substructures in clusters consists of analysing the distribution (both in projected position and velocity) of their brightest blue members in a first step, and then verifying their gas content. This method has successfully revealed substructures at different merging stages in other systems (see, for example, Dickey 1997; Bravo-Alfaro et al. 2000; van Gorkom et al. 2003). We expect that spirals already undergoing strong environmental effects present different degrees of H I deficiency, while the newcomers to the cluster neighborhood display normal gas content. In order to apply this method to A 85 we built a catalogue of the brightest blue member galaxies $^{3}$ based on the following strategy. (1) Magnitude and color: taking superCOSMOS data we produce a color-magnitude diagram to exclude the red sequence. In this fashion we select all galaxies brighter than $b_{\mathrm{J}}=18.0$ and bluer than $b_{\mathrm{J}}-r_{\mathrm{F}}=1.2$. (2) Position: we take only the objects within the reported region surveyed in HI, as shown in Fig. 5. (3) Velocity: we only consider galaxies with known redshift, and within the effective H I velocity coverage $14600-18400 \mathrm{~km} \mathrm{~s}^{-1}$; this represents a velocity interval roughly centered on the systemic value for A 85 $\left(16600 \mathrm{~km} \mathrm{~s}^{-1}\right)$ and encompassing roughly three times the cluster velocity dispersion.

There are 42 galaxies matching these criteria which constitute our sample of the brightest blue members of A 85; they are listed in Table 1. After the running number (first column), the next three columns list galaxy names and positions. Galaxies with an asterisk were detected in $\mathrm{HI}$ and have reported gas masses of over $10^{9} M_{\odot}$. The symbol "\#” indicates galaxies with strong $\mathrm{H} \alpha$ emission (as detected by Boué et al. 2008), and "\#\#" indicates a high probability of harboring an AGN, according to coincident positions with X-ray point-like detections by Sivakoff et al. (2008). A particular case is the blue H Irich galaxy J0043017-094721 which, due to its projected position very close to A85[DFL98]425, had remained previously uncatalogued as an independent source. These two objects are roughly coincident with the position that Abell et al. (1989) reported for the cluster A 87, but are separated in velocity by more than $10000 \mathrm{~km} \mathrm{~s}^{-1}$. The position of J0043017-094721 was

\footnotetext{
3 In the absence of morphological classification, we expect that bright blue objects will very likely be late-type galaxies.
} 
Table 1. The sample of brightest blue member galaxies of A 85. Objects with an asterisk are H I detections. “\#” indicates strong H $\alpha$ emission, and “\#\#” indicates high probability of an AGN (see text).

\begin{tabular}{|c|c|c|c|c|c|c|c|c|}
\hline $\begin{array}{r}\text { Running } \\
\text { number } \\
\text { (1) }\end{array}$ & Name & $\alpha_{2000}$ & $\delta_{2000}$ & $\begin{array}{c}v_{\mathrm{opt}} \\
\left(\mathrm{km} \mathrm{s}^{-1}\right) \\
(5)\end{array}$ & $\begin{array}{r}\Delta v_{\text {opt }} \\
\left(\mathrm{km} \mathrm{s}^{-1}\right) \\
(6)\end{array}$ & (7) & $\begin{array}{c}b_{\mathrm{J}}-r_{\mathrm{F}} \\
(8)\end{array}$ & $\begin{array}{r}\text { Dist } \\
(\mathrm{Mpc})\end{array}$ \\
\hline 1 & A85[DFL98] 079* & 04031.66 & -091320.0 & 15480 & 121 & 17.492 & 1.016 & 1.35 \\
\hline 2 & A85[DFL98] 133 & 04112.80 & -09 3204.0 & 17156 & 114 & 16.547 & 1.028 & 1.12 \\
\hline 3 & A85[DFL98] 139* & 04114.19 & -085553.4 & 15146 & 185 & 17.978 & 0.593 & 1.60 \\
\hline 4 & A85[DFL98] 150\# & 04119.82 & -092327.0 & 14728 & 125 & 17.649 & 1.104 & 0.62 \\
\hline 5 & A85[DFL98] 167 & 04127.16 & -091343.1 & 14203 & 147 & 16.802 & 1.080 & 0.49 \\
\hline 6 & A85[DFL98] 178 & 04130.75 & -0902 14.9 & 15231 & 116 & 17.210 & 1.092 & 1.11 \\
\hline 7 & A85[DFL98] 193 & 04134.91 & -090047.5 & 17550 & 88 & 17.720 & 1.179 & 1.19 \\
\hline 8 & A85[DFL98] 201 & 04136.22 & -08 5934.7 & 17969 & 174 & 17.432 & 1.121 & 1.26 \\
\hline 9 & A85[DFL98] 221\#\# & 04142.96 & -092621.4 & 16854 & 143 & 15.523 & 1.187 & 0.56 \\
\hline 10 & A85[DFL98] 226\# & 04145.44 & -094033.3 & 17063 & 159 & 17.783 & 0.961 & 1.49 \\
\hline 11 & J0041490-095705 & 04149.00 & -095704.6 & 15536 & 90 & 16.860 & 0.968 & 2.59 \\
\hline 12 & A85[DFL98] 255\# & 04153.45 & -09 2940.8 & 15326 & 115 & 15.757 & 0.528 & 0.77 \\
\hline 13 & A85[DFL98] 267 & 04157.34 & -09 3523.9 & 15885 & 144 & 17.554 & 1.025 & 1.15 \\
\hline 14 & J0041599-093910 & 04159.89 & -09 3910.0 & 15761 & 113 & 16.330 & 0.833 & 1.41 \\
\hline 15 & A85[DFL98] 279 & 04202.16 & -09 4017.6 & 14097 & 147 & 17.803 & 0.905 & 1.48 \\
\hline 16 & A85[DFL98] 286 & 04205.04 & -093203.9 & 15832 & 168 & 15.959 & 0.969 & 0.96 \\
\hline 17 & A85[DFL98] 291\# & 04206.01 & -093606.5 & 15865 & 117 & 17.476 & 1.198 & 1.22 \\
\hline 18 & A85[DFL98] 300 & 04210.28 & -085551.6 & 17670 & 154 & 17.566 & 1.176 & 1.52 \\
\hline 19 & A85[DFL98] 306 & 04211.91 & -095254.9 & 16296 & 125 & 17.160 & 1.114 & 2.34 \\
\hline 20 & A85[DFL98] 316 & 04217.27 & -092847.7 & 15863 & 103 & 17.554 & 1.073 & 0.84 \\
\hline 21 & A85[DFL98] 323* & 04218.72 & -095413.5 & 15577 & 117 & 17.967 & 0.167 & 2.45 \\
\hline 22 & A85[DFL98] 338 & 04224.23 & -091616.6 & 18184 & 115 & 17.237 & 1.080 & 0.58 \\
\hline 23 & A85[DFL98] 347* & 04229.47 & -100106.9 & 15191 & 85 & 17.750 & 0.595 & 2.93 \\
\hline 24 & A85[DFL98] 356 & 04233.76 & -085253.5 & 17780 & 135 & 17.002 & 1.178 & 1.83 \\
\hline 25 & A85[DFL98] 358 & 04233.91 & -090846.0 & 16685 & 157 & 17.351 & 1.048 & 0.96 \\
\hline 26 & A85[DFL98] 363 & 04234.75 & -093918.1 & 16363 & 112 & 15.873 & 1.188 & 1.59 \\
\hline 27 & A85[DFL98] 374* & 04241.47 & -085649.0 & 15149 & 148 & 16.586 & 0.907 & 1.66 \\
\hline 28 & A85[DFL98] 382\# & 04243.91 & -094420.7 & 15232 & 119 & 17.296 & 1.149 & 1.96 \\
\hline 29 & A85[DFL98] 391 & 04248.38 & -093440.9 & 17934 & 175 & 17.885 & 0.945 & 1.46 \\
\hline 30 & J0043017-094721* & 04301.71 & -094734.3 & 15110 & 22 & 17.411 & 0.269 & 2.29 \\
\hline 31 & A85[DFL98] 429 & 04304.16 & -093243.1 & 15846 & 156 & 17.499 & 1.159 & 1.56 \\
\hline 32 & A85[DFL98] 435 & 04306.00 & -095014.4 & 14767 & 135 & 16.953 & 1.196 & 2.48 \\
\hline 33 & A85[DFL98] 447 & 04310.91 & -094053.9 & 16489 & 72 & 16.110 & 1.143 & 2.02 \\
\hline 34 & A85[DFL98] 451\#\# & 04311.59 & -093816.2 & 16352 & 103 & 16.024 & 1.054 & 1.90 \\
\hline 35 & A85[DFL98] 461* & 04314.34 & -09 1021.4 & 15008 & 142 & 19.005 & 0.444 & 1.49 \\
\hline 36 & A85[SDG98] 3114* & 04319.56 & -09 0912.3 & 15060 & 22 & 19.360 & 0.285 & 1.60 \\
\hline 37 & A85[DFL98] 483 & 04330.48 & -094359.4 & 15157 & 151 & 17.309 & 1.087 & 2.39 \\
\hline 38 & A85[DFL98] 486* & 04331.16 & -095146.4 & 16582 & 94 & 17.044 & 0.564 & 2.79 \\
\hline 39 & A85[DFL98] 491* & 04334.04 & -085037.0 & 14989 & 130 & 17.164 & 0.559 & 2.52 \\
\hline 40 & A85[DFL98] 494 & 04336.31 & -092547.7 & 15051 & 195 & 16.830 & 1.168 & 1.83 \\
\hline 41 & A85[DFL98] 496* & 04338.77 & -09 3120.6 & 15068 & 130 & 17.165 & 0.504 & 2.00 \\
\hline 42 & A85[DFL98] 502* & 04343.98 & -090422.9 & 14830 & 128 & 17.694 & 0.772 & 2.10 \\
\hline
\end{tabular}

determined in this work from a MEGACAM image taken at the 3.6-m CFHT (Boué et al. 2008). In Col. 5 we give the optical radial velocity of the galaxies, obtained from the compilation described in Sect. 2. The respective estimated error (rms) in velocity is given in Col. 6. Column 7 gives the $b_{\mathrm{J}}$ SuperCOSMOS magnitude of the galaxies. Column 8 gives the $b_{\mathrm{J}}-r_{\mathrm{F}}$ color. Column 9 gives the projected distance to the cluster center in Mpc.

One of the most important results of this paper is shown in Fig. 5, where a very striking asymmetry in the blue-galaxy distribution through the whole cluster volume is seen. Crosses indicate the 42 brightest blue (cluster-member) galaxies plotted over a composite view of A 85 seen in optical and X-rays. As expected, the $12 \mathrm{HI}$ detections (encircled crosses) are within this sample, and the remaining 30 constitute the category of gas deficient (H I-non detected) blue galaxies. To check if this asymmetric distribution is really a property of the blue members of A 85, we examined the distribution of non-emission line cluster galaxies taken from a sample of 232 objects with SDSS spectra, which should be representative of red members. As expected, these display a very regular distribution, concentrated around the cluster center (Caretta et al. 2008).

The spatial distribution of the $\mathrm{H}$ I detections follows the same asymmetric trend as blue galaxies: all but two are projected east of the center of A 85 where they are roughly distributed in two regions. The first group of five $\mathrm{HI}$ detections is projected at an average distance of $\sim 30^{\prime} \mathrm{NE}$ from the center of A 85. An interesting feature to notice is that these five $\mathrm{HI}$-rich blue galaxies (A85[DFL98]374/461/491/502 and [SDG98]3114) display an intriguingly low velocity dispersion of just $120 \mathrm{~km} \mathrm{~s}^{-1}$, but are spread (in projection) across a large area of $\sim 1.2 \mathrm{Mpc}$.

The second group of $\mathrm{HI}$ detections is projected SE of A 85, around the position of our detected $S E$ substructure (see Fig. 4). Five galaxies are detected in $\mathrm{HI}$ in this region with at least four of them having higher probabilities of belonging to a real subcluster when compared to those in the NE (see below). In 


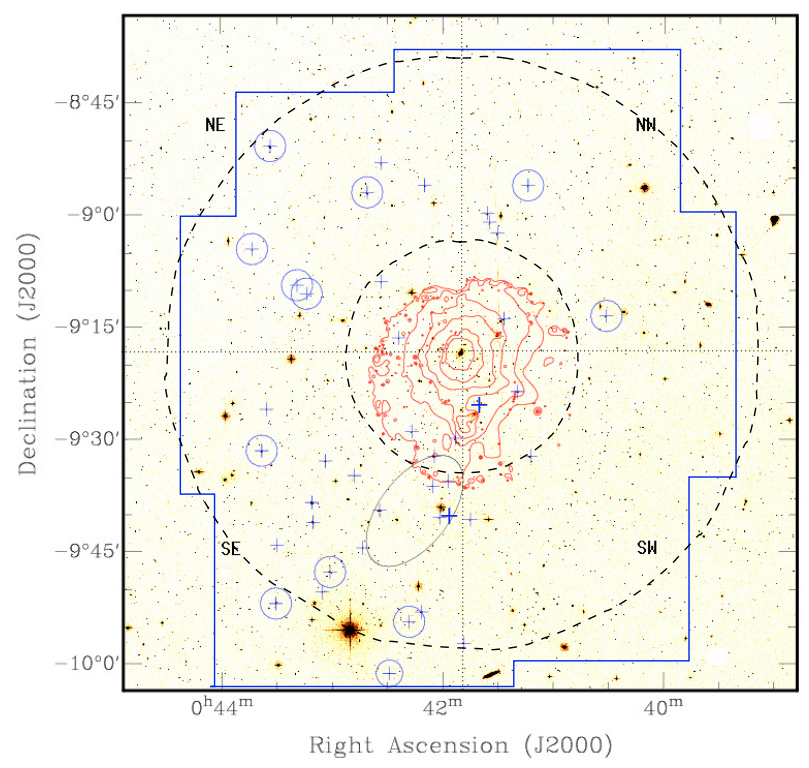

Fig. 5. Distribution of the brightest blue galaxies through A85, indicating the position of the $\mathrm{H}$ I rich (encircled crosses) and the H I poor galaxies (crosses). The center of the X-ray emission (red contours) as seen by ROSAT coincides with the $\mathrm{cD}$ galaxy. The ellipse roughly draws the position of the X-ray filament mentioned in the text. Large dashed circles correspond to the $1.0 \mathrm{Mpc}$ and $2.4 \mathrm{Mpc}$ radius. The blue external polygon indicates the VLA-H I surveyed area.

addition to these H I detections (A85[DFL98]323, 486, 347 and J004301-094721), many other (gas poor) blue objects appear in the SE quadrant. The H I-rich galaxies are projected, on average, some $35^{\prime} \mathrm{SE}$ of the center of A 85, along the extension of the $\mathrm{X}$-ray filament (indicated by an ellipse in the figure). Similar to the five galaxies detected NE of A 85, these are also spread over a large area (more than one Mpc, Bravo-Alfaro et al. 2008), and they form a grouping with low velocity dispersion (see Table 1).

The asymmetry in the distribution of blue galaxies is better quantified if we refer to the quadrants indicated in the figure. $\mathrm{SW} \rightarrow \mathrm{NW} \rightarrow \mathrm{NE} \rightarrow \mathrm{SE}$ gives the sequence from the poorest to the richest in blue galaxies. Relative to the other quadrants, the $\mathrm{SW}$ region is almost empty of blue galaxies, containing only four of these objects (all H I-poor). In comparison, six blue objects are seen in the NW and nine in the NE, where two and five $\mathrm{HI}$ detections were found respectively. By far the richest region in blue galaxies is the SE, where 23 blue objects are distributed from the highest density zone of the cluster ICM, going through the X-ray filament area (indicated by an ellipse), and then spread around the position of the $S E$ substructure. Only five of those are detected in HI. Most of the blue galaxies projected on this region display velocities in the range $15000-16300 \mathrm{~km} \mathrm{~s}^{-1}$ (Table 1), and several display wide $\mathrm{H} \alpha$ equivalent widths (larger than $25 \AA$ ). The presence of this indicator of star formation (Boué et al. 2008, and Paper II in prep.) strongly supports the hypothesis of a merging between a subcluster of galaxies and A 85 .

\section{Discussion}

\subsection{Substructures across $A 85$}

The results of the previous section clearly reveal the following substructures.

The core: the central region of the cluster appears to be dynamically very active and presents strong signs of past activity
(Kempner et al. 2002; Durret et al. 2005). We confirm this by detecting the most prominent substructure $(C 2)$ projected $\sim 5^{\prime}$ west of the cluster center and consisting of two blobs. The ridge defined by these two blobs is oriented in a SE-NW axis, and the position angle of this axis is roughly the same as the orientation of the X-ray filament and its extension to the $S E$ substructure. Most of the galaxies in $C 2$ present velocities around $15800 \mathrm{~km} \mathrm{~s}^{-1}$, well under the systemic value of A $85\left(16600 \mathrm{~km} \mathrm{~s}^{-1}\right)$.

The southern part of $C 2$ has been seen in X-rays as a diffuse source with Chandra (see Fig. 1 of Kempner et al. 2002, the structure which they call "the southwest subcluster"). Here we report that the clump of galaxies associated with this structure further extends some $10^{\prime}$ to the $N$ (see Fig. 4), with another clump centered on the second brightest galaxy of A 85 (KAZ 364). This is a very peculiar spiral reported as an individual X-ray source by Sivakoff et al. (2008), and classed an AGN. In spite of the large area covered by this survey, the authors only report individual X-ray sources; no diffuse emission is detected around KAZ 364, equivalent to that seen by Kempner et al. (2002) in the southern tail of this grouping. This object also has the lowest radial velocity in the whole A 85 sample and, being outside the VLA velocity coverage, it is not included in Table 1 . The $\delta$ signals associated with $C 2$ decreases if we remove this galaxy from the sample, but the difference is not significant. The whole $C 2$ structure, with diffuse $\mathrm{X}$-ray emission on its southern tail, strongly suggests a subcluster undergoing an advanced merging process with the main cluster core, in agreement with the general scenario proposed by Durret et al. (2005).

The southern blob and filament: region $S B$, projected some $10^{\prime}$ south of the $\mathrm{cD}$, probably consists of an almost disrupted group of galaxies merging from the south (Kempner et al. 2002). Intriguingly enough, the galaxies within this structure display velocity values around $17000 \mathrm{~km} \mathrm{~s}^{-1}$, i.e. larger than the substructures $C 2, F$ and $S E$. Clearly identified in Fig. 3, this substructure has the most accentuated density enhancement, even comparable to the core of A 85, while its kinematics do not deviate much from the global pattern - this is indicated with small green circles in Fig. 4 at the same position. The opposite occurs with clump $F$, a substructure projected midway along the X-ray filament described by Durret et al. (2003), about 20' away from the cluster center. This is not detected as a significant enhancement in the isodensity plot (Fig. 3) but deviates very much from the global kinematics (see the large black circles in Fig. 4). This structure is between the subgroup 3 in Durret et al. (1998b) and the southern blob.

The SE region: as outlined in the previous sections, the $\mathrm{SE}$ is a very interesting region. The substructure marked $S E$ in Fig. 4 is close to the projected position of the A 87 cluster, roughly in the middle of the X-ray filament as detected in the ROSAT PSPC field (Durret et al. 1998b), or equivalently, at the end of the filament portion detected in the XMM-Newton field (Durret et al. 2003). This is a clump with very prominent kinematic deviations (large black circles) but is barely detected as a galaxy density enhancement in Fig. 3. The most likely explanation is that we are dealing with a relatively poor subcluster undergoing a high velocity infall towards A 85. Many of the galaxies within this substructure display velocities around $15800 \mathrm{~km} \mathrm{~s}^{-1}$, the same as substructures $F$ and $C 2$. Most of the $S E$ galaxies have blue colors, indicative of late type morphologies, and the galaxies detected in H I southeast of A 85, being within this velocity range, are certainly associated with this infalling group. In Paper II we analyse the spectral properties and NIR images of selected galaxies in this area. 
The W group: another region with several large $\delta$ values is seen about $35^{\prime}$ west of the cluster center (marked with $W$ ). It is roughly at the same projected distance from the cluster center as the $S E$ clump. These objects are outside the useful VLA-FOV and we have no information on their gas content; furthermore it is outside all the reported X-ray surveys. This group of galaxies displays a velocity around $18000 \mathrm{~km} \mathrm{~s}^{-1}$. As this group is not projected along a major large scale filament, it is impossible to say if a dynamical relation exists with A 85 .

The NE region: the $\mathrm{HI}$-detected galaxies in the NE quadrant of A 85 (encircled crosses in Fig. 3), in spite of displaying a tight velocity dispersion, are neither detected in the isodensity plot (Fig. 3) nor as a kinematical structure (Fig. 4). We applied larger values of $N_{\text {neighb }}$ unsuccessfully trying to separate these objects from the global kinematics. This is likely due to the very large area over which they are spread and to the small number of galaxies being part of this possible structure.

\subsection{HI-rich vs. HI-poor galaxies: the radial distribution}

Striking observational evidence found in many of the clusters surveyed in $\mathrm{HI}$ is the presence of galaxies and groups, projected at large radius from the cluster center, that have been stripped of a major fraction of their gas component (e.g. Solanes et al. 2001, and references therein). Their large distance from the densest zones of the ICM raises the question of whether the typical ram pressure stripping approach could account for such effects. The best known examples have been reported in Coma (Gavazzi 1987; Bravo-Alfaro et al. 2000) and in Perseus I (Levy et al. 2007). Based on Arecibo data, Gavazzi (1987) reported a number of H I deficient galaxies at large radii from Coma, projected on the intermediate zone between this cluster and A 1367. In addition to this, Bravo-Alfaro et al. (2000) reported one group of late-type galaxies projected $0.9 \mathrm{Mpc}$ east of the Coma center, with strong H I deficiency. More recently, Levy et al. (2007) found a very interesting picture in Pegasus I, a system which lacks a harsh ICM and where we do not expect ram pressure to strip large amounts of gas. These authors report a number of spirals, near the cluster core but also in foreground and background groups, ranging from moderate H I deficiency to truncated gas disks. These cases can be added to other stripped objects in low density environments, such as NGC 4522 in Virgo (Kenney et al. 2004, and references therein) and NGC 2276 in the NGC 2300 group (Davis et al. 1997). With the same trend we have A 85 , with many gas-poor blue galaxies lying at $2-2.5 \mathrm{Mpc}$ from the cluster center in projection.

A very important step forward on this issue was made by Tonnesen et al. (2007), who carried out a cosmological simulation of cluster evolution. They found that gas stripping may occur out to the cluster virial radius, as observed in A 85 and the clusters mentioned above. Very interestingly, their model predicts that ram pressure stripping remains the most important mechanism responsible for the loss of inter-stellar gas, and they found that a fraction of galaxies is affected by this process in the intermediate cluster zone $(1.0-2.4 \mathrm{Mpc})$ and up to the periphery $(2.4-5.0 \mathrm{Mpc})$. This is in close agreement with our observational results. With the help of Table 1 and Fig. 5 we see that, among the 42 brightest blue member galaxies of A 85, 30 are deeply gas deficient and constitute our sample of blue gas-poor galaxies. The radial distribution of these $\mathrm{HI}$-deficient objects is as follows: only 8 are within the inner radius $(r<1.0 \mathrm{Mpc})$ while 22 are in the transition zone $(1.0-2.4 \mathrm{Mpc})$. These gas-poor blue

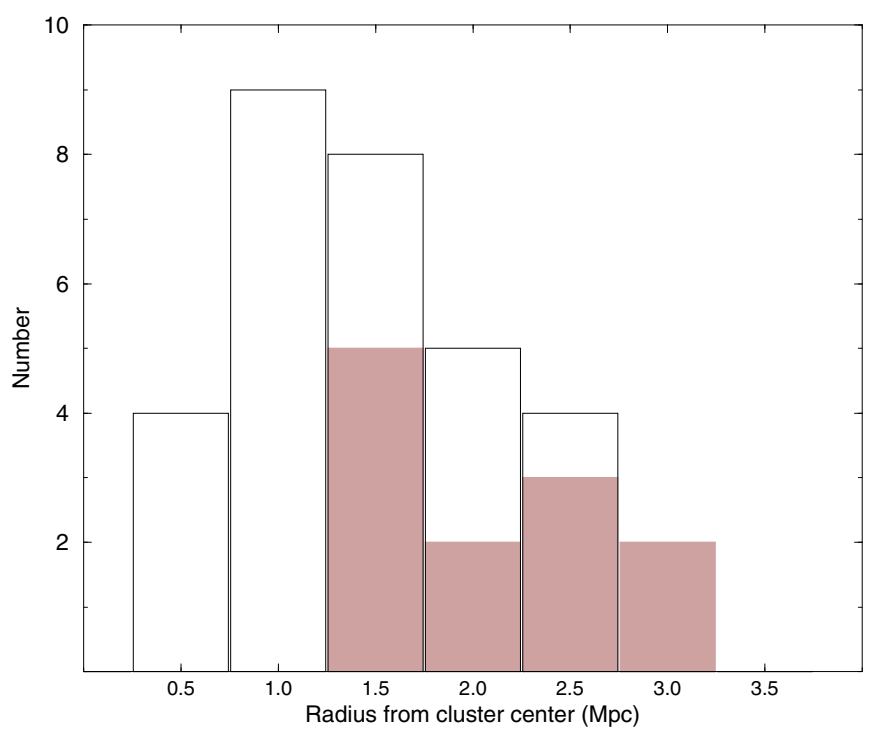

Fig. 6. Histogram of the radial distribution of the two populations: H I-rich (gray columns) vs. H I-poor (empty columns) galaxies. This clearly shows that, on average, the former are found at larger distances from the cluster center.

galaxies along the transition zone of A 85 would match the stripped population predicted by Tonnesen et al. (2007).

Figure 6 clearly shows that projected positions of $\mathrm{H}$ I-rich galaxies (filled columns), measured from the cluster center, follow a different distribution when compared with the H I-non detections (empty columns, see also Table 1). The histogram displays bins of $0.5 \mathrm{Mpc}$, where the $\mathrm{X}$-axis corresponds to the radius measured from the $\mathrm{cD}$ galaxy. Average positions are 2.07 Mpc and 1.42 Mpc, for H I-rich and H I-deficient galaxies, with standard deviations of 0.53 and 0.59 respectively. In order to examine the difference between both distributions we applied the Kolmogorov-Smirnov test, which is sensitive to global shape differences. We find they are different with a significance level of 0.99 . We complement this by applying the $t$ distribution to explore the difference of two means (case $n_{1}+n_{2}-2=40$ degrees of freedom). The hypothesis $H_{0}: \mu_{1}=\mu_{2}$ is discarded with a confidence level of 0.99 .

\subsection{Ram pressure stripping in $A 85$}

In order to quantify the strength of the ICM-ISM interaction exerted on our sample of blue galaxies around A 85 we compute, as a first approach, the cluster gas density distribution and extrapolate it to radii of $2.0-2.5 \mathrm{Mpc}$. We follow the hydrostatic-isothermal $\beta$-model of Cavaliere \& Fusco-Femiano (1976), given by:

$\rho(r)=\rho_{o}\left[1+\left(r / r_{\mathrm{c}}\right)^{2}\right]^{-3 \beta / 2}$.

We take the parameters of the gas profile of A 85 from Chen et al. (2007): core radius $r_{\mathrm{c}}=82 \mathrm{kpc}$, central density $\rho_{o}=0.0257 \mathrm{~cm}^{-3}$ (and assuming that this is an electrically neutral gas), and $\beta=$ 0.532 . We then compute the ram pressure stripping as a function of radius from the cluster center following Gunn \& Gott's (1972) equation: $P_{\text {ram }}=\rho_{\text {ICM }} \times v_{\text {rel }}^{2}$, where $\rho_{\text {ICM }}$ is the density of the ICM at the galaxy position, and $v_{\text {rel }}$ is the galaxy velocity relative to the ICM. Ideally, the latter should be the component of the real velocity perpendicular to the galaxy disk (as defined 
e.g. in Cayatte et al. 1994), but not having information on the inclination angle nor on the velocity of the galaxy in the plane of the sky we will thus consider radial velocities, relative to the cluster systemic movement, as a first approach. Normally, since galaxy tangential velocity components are expected, this approximation underestimates the real $v_{\text {rel }}$, making our value of $P_{\text {ram }}$ a lower limit of the real one.

For the estimation of $P_{\text {ram }}(r)$ we consider three values of $v_{\text {rel }}$ : first we take $1000 \mathrm{~km} \mathrm{~s}^{-1}$, roughly matching the (radial) velocity dispersion of the cluster, which is also a lower limit of the (radial) relative velocity between the $S E$ clump $\left(\sim 15800 \mathrm{~km} \mathrm{~s}^{-1}\right)$ and A $85\left(\sim 16600 \mathrm{~km} \mathrm{~s}^{-1}\right)$. We include two other velocity values as reference, $500 \mathrm{~km} \mathrm{~s}^{-1}$ and $1500 \mathrm{~km} \mathrm{~s}^{-1}$. Figure 7 displays the output of $P_{\text {ram }}$ for values between 1.0 and $2.6 \mathrm{Mpc}$.

In order to quantify the effects of ram pressure stripping on individual galaxies we compare these values with the restoring force, defined as $F_{\mathrm{r}}=2 \pi G \sigma_{\text {tot }} \sigma_{\text {gas }}$. This is a measure of the galaxy autogravitational binding force per $\mathrm{cm}^{-2}$ (having units of dyn $\mathrm{cm}^{-2}$, it can be directly compared with the ram pressure). For this comparison we take the typical value of $F_{\mathrm{r}}=$ $2 \times 10^{-12}$ dyn $\mathrm{cm}^{-2}$, obtained by Cayatte et al. (1994) for spiral galaxies in Virgo. If we inspect Fig. 7 we notice that, in spite of the low ICM density values in the transition zone (where the $S E$ clump is found), $P_{\text {ram }}$ is larger than the restoring force up to $2.0 \mathrm{Mpc}$ from the cluster center for $v_{\text {rel }} \geq 1000 \mathrm{~km} \mathrm{~s}^{-1}$. Even the lowest velocity in our estimation $\left(v_{\mathrm{rel}}=500 \mathrm{~km} \mathrm{~s}^{-1}\right)$ produces values of ram pressure of the same order of magnitude as $F_{\mathrm{r}}$ within the inner $1 \mathrm{Mpc}$. This unexpected result confirms that we should not discard ram pressure stripping as a mechanism playing a major role in driving galaxy evolution when dealing with high infall velocities relative to a cluster with a dense ICM such as that of $\mathrm{A} 85$.

\section{Summary}

Our results can be summarized as follows:

1. We first analysed the distribution of galaxies with measured redshift in the line of sight towards the complex A 85/87/89, within a region of about 1.5 degrees side around the center of A 85. For A 85 we find 367 member galaxies in the velocity range $13000-20000 \mathrm{~km} \mathrm{~s}^{-1}$. We confirm the presence of two background structures near the position of A 89. The farthest cluster in our sample is A 87, at $\sim 39000 \mathrm{~km} \mathrm{~s}^{-1}$.

2. We revisited the $2 \mathrm{D}$ (RA, Dec) distribution of galaxies through the whole A 85/87/89 complex and trace for the first time the isodensity contour map only with members of A 85 . This highlights the substructures of A 85 from the background clusters. Three major density peaks are revealed: two in the inner part of the cluster and one at $\sim 2 \mathrm{Mpc}$ in the SE direction, superimposed on the projected position of A 87 .

3. We ran the $3 \mathrm{D}$ kinematical $\Delta$-test (RA, Dec, vel) for the member galaxies of A 85. We detect different clumps of galaxies going from the center of A 85 and extending towards the SE. The first substructure reveals the inner structure of the core of A 85, with a group of galaxies in an advanced merging stage with the main cluster body. We also report a structure coincident with the X-ray Southern Blob, roughly $0.7 \mathrm{Mpc}$ south of the center of A 85. Another substructure appears along the X-ray filament, more than $1 \mathrm{Mpc} \mathrm{SE}$ of the cluster center. Finally, the $S E$ clump is projected at more than $2 \mathrm{Mpc}$ from the center of A 85, at $\sim 15800 \mathrm{~km} \mathrm{~s}^{-1}$, coincident with the infalling sub-cluster revealed by the $2 \mathrm{D}$ analysis.

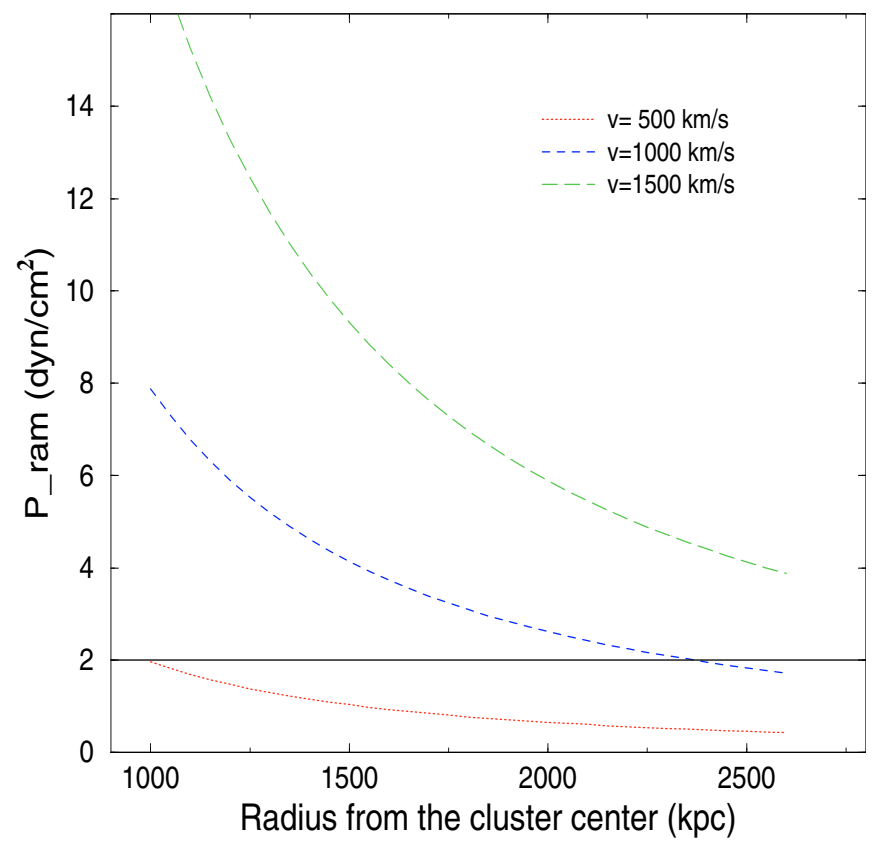

Fig. 7. The ram pressure stripping, in units of $10^{-12} \mathrm{dyn} \mathrm{cm}^{-2}$, as a function of distance from the center of A 85, estimated for radii up to $\sim 2.5 \mathrm{Mpc}$. Three different velocities, relative to the ICM, were applied: 500,1000 and $1500 \mathrm{~km} \mathrm{~s}^{-1}$. As a comparison, the solid line indicates the value of the restoring force $F_{\mathrm{r}}$ (see text).

4. We built a sample of the brightest blue galaxies, with 42 members of A 85 in the velocity range 14600-18400 $\mathrm{km} \mathrm{s}^{-1}$, and we trace their spatial distribution throughout the cluster. By taking into account the region surveyed in $\mathrm{HI}$ and the velocity range covered by Bravo-Alfaro et al. (2008), we obtain information on the gas content of this sample, with just twelve objects (H I-detections) showing rather normal gas content or mild deficiency; the remaining 30 blue objects represent deficient galaxies. The spatial distribution of the whole 42 blue brightest members is totally unexpected, with about half of them projected on the SE quadrant of the cluster. This strongly supports the presence of sub-clusters in an early stage of merging with A 85, infalling with a relative velocity greater than $1000 \mathrm{~km} \mathrm{~s}^{-1}$.

5. The distribution of the blue sample is also remarkable when measured as a function of the projected distance to A 85 . From the sample of 42 blue members, 32 are projected on the transition zone (1.0-2.4 Mpc), and 22 of these are very gas deficient. We compare the positions of the two populations as a function of projected radius from the cluster center. We show that H I-rich galaxies are projected, on average, much further than H I-deficient ones: the mean projected distances are $2.1 \mathrm{Mpc}$ and $1.4 \mathrm{Mpc}$ respectively.

6. We estimated ram pressure stripping in A 85 as a function of radius in order to quantify the environment's ability to decrease the gas fraction in spirals, in particular in the gas-poor blue galaxies reported in the SE region, $\sim 2 \mathrm{Mpc}$ away from the center of A 85. Considering gas density parameters of A 85 ( $\beta$ model and X-ray parameters) and different values of velocity relative to the ICM, we found that ram pressure is several times larger than the restoring force for typical spirals, when velocities relative to A 85 are over $1000 \mathrm{~km} \mathrm{~s}^{-1}$. This would explain the presence of the impressive sample of gas-poor blue-galaxies projected at the edge of the 
transition zone, i.e. at radius $\gtrsim 2 \mathrm{Mpc}$. The observational evidence shown here matches theoretical cluster models such as that of Tonnesen et al. (2007) who predict active gas stripping in that transition zone.

These results reveal an even more complex structure of A 85 than previously found, with groups of galaxies at different merging stages, especially in the SE region but in the cluster core as well. The observational evidence shown in this work confirms that the cluster environment is playing an important role in driving galaxy evolution. We show that ram pressure stripping might be effective up to unusually large radial distances from a cluster center, when infalling velocities are large enough. In a forthcoming paper (Paper II) we will quantify the effects of gravitational mechanisms in disturbing stellar disks, in particular in the SE region.

Acknowledgements. We thank the anonymous referee for her/his very useful comments which helped us to strenghten and improve this paper. The authors are also grateful to $\mathrm{H}$. Andernach and $\mathrm{E}$. Tago for providing radial velocity data from their compilation. H.B.A. acknowledges CONACyT grant 50794, and financial support from Institut d'Astrophysique de Paris and Centro de Astrofísica da Universidade do Porto, allocated to carry out working stays at these institutes. C.A.C. also aknowledges support from CONACyT, grant 50921, and the University of Guanajuato (DINPO) for the grant 0102/06. C. Lobo aknowledges finantial support from project PTDC/CTE-AST/66147/2006 (FCT, Portugal).

\section{References}

Abell, G. O., Corwin, Jr., H. G., \& Olowin, R. P. 1989, ApJS, 70, 1

Adami, C., Biviano, A., Durret, F., \& Mazure, A. 2005, A\&A, 443, 17

Andernach, H., Tago, E., Einasto, M., Einasto, J., \& Jaaniste, J. 2005, in Nearby Large-Scale Structures and the Zone of Avoidance, ed. A. P. Fairall, \& P. A. Woudt, ASP Conf. Ser., 329, 283

Arnouts, S., de Lapparent, V., Mathez, G., et al. 1997, A\&AS, 124, 163

Arnouts, S., Vandame, B., Benoist, C., et al. 2001, A\&A, 379, 740

Beers, T. C., Flynn, K., \& Gebhardt, K. 1990, AJ, 100, 32

Beers, T. C., Gebhardt, K., Forman, W., Huchra, J. P., \& Jones, C. 1991, AJ, 102, 1581

Boué, G., Durret, F., Adami, C., et al. 2008, A\&A, 489, 11

Bravo-Alfaro, H., Cayatte, V., van Gorkom, J. H., \& Balkowski, C. 2000, AJ, 119,580

Bravo-Alfaro, H., van Gorkom, J. H., \& Caretta, C. 2008, in Practical Cosmology Vol. I, Proc. of the int. conf. Problems of Practical Cosmology, ed. Y. V. Baryshev, I. N. Taganov, \& P. Teerikorpi, 102
Caretta, C. A., Islas, J. M., Torres-Papaqui, J. P., et al. 2008, MSAIt, 79 Cavaliere, A., \& Fusco-Femiano, R. 1976, A\&A, 49, 137

Cayatte, V., van Gorkom, J. H., Balkowski, C., \& Kotanyi, C. 1990, AJ, 100, 604

Cayatte, V., Kotanyi, C., Balkowski, C., \& van Gorkom, J. H. 1994, AJ, 107, 1003

Chen, Y., Reiprich, T. H., Böhringer, H., Ikebe, Y., \& Zhang, Y.-Y. 2007, A\&A, 466, 805

Christlein, D., \& Zabludoff, A. I. 2003, ApJ, 591, 764

Chung, A., van Gorkom, J. H., Kenney, J. D. P., \& Vollmer, B. 2007, ApJ, 659, L115

Davis, D. S., Keel, W. C., Mulchaey, J. S., \& Henning, P. A. 1997, AJ, 114, 613

Dickey, J. M. 1997, AJ, 113, 1939

Dressler, A. 1980, ApJ, 236, 351

Dressler, A. 2004, in Clusters of Galaxies: Probes of Cosmological Structure and Galaxy Evolution, ed. J. S. Mulchaey, A. Dressler, \& A. Oemler, 206

Dressler, A., \& Shectman, S. A. 1988, AJ, 95, 985

Durret, F., Felenbok, P., Lobo, C., \& Slezak, E. 1998a, A\&AS, 129, 281

Durret, F., Forman, W., Gerbal, D., Jones, C., \& Vikhlinin, A. 1998b, A\&A, 335, 41

Durret, F., Lima Neto, G. B., Forman, W., \& Churazov, E. 2003, A\&A, 403, L29 Durret, F., Lima Neto, G. B., \& Forman, W. 2005, A\&A, 432, 809 Gavazzi, G. 1987, ApJ, 320, 96

Giovannini, G., Tordi, M., \& Feretti, L. 1999, New Astron., 4, 141

Gunn, J. E., \& Gott, J. R. I. 1972, ApJ, 176, 1

Hambly, N. C., Davenhall, A. C., Irwin, M. J., \& MacGillivray, H. T. 2001a, MNRAS, 326, 1315

Hambly, N. C., Irwin, M. J., \& MacGillivray, H. T. 2001b, MNRAS, 326, 1295 Kempner, J. C., Sarazin, C. L., \& Ricker, P. M. 2002, ApJ, 579, 236

Kenney, J. D. P., van Gorkom, J. H., \& Vollmer, B. 2004, AJ, 127, 3361

Levy, L., Rose, J. A., van Gorkom, J. H., \& Chaboyer, B. 2007, AJ, 133, 1104

Li, C., Kauffmann, G., Jing, Y. P., et al. 2006, MNRAS, 368, 21

Lilly, S. J., Le Fevre, O., Hammer, F., \& Crampton, D. 1996, ApJ, 460, L1

Madau, P., Ferguson, H. C., Dickinson, M. E., et al. 1996, MNRAS, 283, 1388

Malumuth, E., Kriss, G., Dixon, W. V. D., Ferguson, H. C., \& Ritchie, C. 1992 , AJ, 104, 495

Oemler, A. J. 1974, ApJ, 194, 1

Pinkney, J., Roettiger, K., Burns, J. O., \& Bird, C. M. 1996, ApJS, 104, 1

Sivakoff, G. R., Martini, P., Zabludoff, A. I., Kelson, D. D., \& Mulchaey, J. S. 2008, ApJ, 682, 803

Smith, R. J., Hudson, M. J., Nelan, J. E., et al. 2004, AJ, 128

Solanes, J. M., Manrique, A., García-Gómez, C., et al. 2001, ApJ, 548, 97

Stoughton, C., Lupton, R. H., Bernardi, M., et al. 2002, AJ, 123, 485

Tonnesen, S., Bryan, G. L., \& van Gorkom, J. H. 2007, ApJ, 671, 1434

van Gorkom, J. H. 2004, in Clusters of Galaxies: Probes of Cosmological

Structure and Galaxy Evolution, ed. J. S. Mulchaey, A. Dressler, \& A. Oemler, 305

van Gorkom, J. H., Bravo-Alfaro, H., Dwarakanath, K. S., et al. 2003, Astrophys. Space Sci., 285, 219

Vollmer, B., Beck, R., Kenney, J. D. P., \& van Gorkom, J. H. 2004, AJ, 127, 3375 